\title{
EL INFORME DEL CONSEJO DE ESTADO DE 15 DE DICIEMBRE DE 2010 SOBRE GARANTÍAS DE CUMPLIMIENTO DEL DERECHO DE LA UNIÓN EUROPEA
}

\author{
JOSÉ ANGEL CAMISÓN YAGÜE \\ Prof. Contratado Doctor de Derecho Constitucional \\ Universidad de Extremadura
}

SUMARIO

I. Introducción.

II. Sobre el incumplimiento del Derecho de la Unión Europea y su procedimiento de sanción: implicaciones para las Comunidades Autónomas.

III. La actual situación del ordenamiento jurídico español ante el incumplimiento del Derecho de la Unión Europea por parte de una Comunidad Autónoma: instrumentos generales de prevención y reacción.

IV. Sobre los mecanismos y procedimientos de repercusión de la responsabilidad por incumplimiento del Derecho de la Unión a las Comunidades Autónomas.

V. Experiencias en Derecho Constitucional comparado.

VI. Propuestas del Consejo de Estado.

VII. Conclusiones. 


\section{INTRODUCCIÓN}

En tanto que la Unión Europea es hoy por hoy fundamentalmente una unión de Es$\operatorname{tados}^{1}$ y su Derecho permanece deliberadamente ajeno ${ }^{2}$ a la forma de organización territorial estatal ${ }^{3}$, la responsabilidad de garantizar el cumplimiento de las obligaciones derivadas del Derecho de la Unión se hace recaer, tal y como prescriben los Tratados, directamente sobre cada uno de los Estados miembros. Esta responsabilidad estatal ante la Unión Europea existe aún cuando la causa que diera origen a su nacimiento pudiera estar claramente identificada en el incumplimiento de uno de los entes políticamente descentralizados integrantes de dicho Estado; pues siempre será el Estado central quien asuma ante la UE, en lugar de dichos entes, la responsabilidad derivada del incumplimiento, que en su caso se produjera ${ }^{4}$. Por este motivo corresponde a los Estados miembros de la Unión, en primer lugar, articular sus propios mecanismos nacionales de garantía del cumplimiento de las obligaciones derivadas del ordenamiento de la Unión que incumben a los entes políticamente descentralizados de dicho Estado. Y, en segundo lugar, determinar si el Estado central puede o no, y en su caso, de qué modo y manera, repercutir sobre el ente territorial causante de la infracción la sanción derivada del incumplimiento que pudieran imponerle las Instituciones comunitarias.

Así, en el concreto caso español, y en tanto que las Comunidades Autónomas tienen atribuido un importante catálogo de competencias directamente vinculadas a competencias residenciadas en la Unión Europea ${ }^{5}$, se producen en ocasiones incumplimientos del Derecho de la UE de los que, aún siendo éstas efectivamente autoras, debe responder el Estado central, en tanto que garante ante la Unión Europea del cumplimiento de las obligaciones derivadas de los Tratados y del resto del ordenamiento comunitario. Esta responsabilidad de garantía del Estado por incumplimientos que pudieran cometer las Comunidades Autónomas tiene en el caso español un doble fundamento jurídico. Por un lado, son los propios Tratados de la Unión quienes señalan al Estado como único res-

1 Vid. en este sentido la Sentencia del Bundesverfassungsgericht de 30 de junio de 2009 sobre la ratificación del Tratado de Lisboa, en la que se califica a la Unión en el actual momento de la integración como una unión de Estados soberanos „Staatenverbund». LÓPEZ CASTILLO, A.; "Alemania en la Unión Europea a la luz de la Sentencia-Lisboa, de 30 de junio de 2009, del Tribunal Constitucional federal alemán» en REDC, $n^{\circ} 87,2009$, págs. 347 y 348 .

2 Art. 4.2 del TUE: «La Unión respetará la igualdad de los Estados miembros ante los Tratados así como su identidad nacional, inherente a las estructuras fundamentales políticas y constitucionales de éstos, también en lo referente a la autonomía local y regional»

3 J.A. MONTILla MARTOS, Derecho de la Unión Europea y de las Comunidades Autónomas, Madrid, CEPC, 2005, pág. 35: «El Derecho de la Unión ha sido tradicionalmente ajeno a la descentralización política de los Estados miembros. Es la conocida ceguera federal comunitaria (Landesblidheit)...».

4 Se mantiene así en la UE, y aún a pesar del significativo grado de integración política existente entre los Estados miembros, un principio típico del Derecho Internacional Público por el que la violación de un Tratado por parte de un órgano del Estado o de una entidad pública territorial integrante del mismo, se hace recaer sobre el Estado. Vid. F. RUIZ RUIZ, «La función de garantía del cumplimiento autonómico del Derecho comunitario Europeo» en REDC, $\mathrm{n}^{\circ} 51,1997$, págs. 160-161

5 Debe recordarse que, tal y como ha establecido reiteradamente la jurisprudencia del TC, las competencias relativas al desarrollo y ejecución del Derecho de la Unión corresponden a la Administración que materialmente ostente la competencia según las reglas de Derecho nacional. Vid. en este sentido el FJ 1 de la STC $79 / 1992$ de 28 de mayo de 1992. 
ponsable jurídico ${ }^{6}$. Por otro lado, el art. 93 de la Constitución atribuye al Estado central, y concretamente a las Cortes Generales o al Gobierno, según los casos, la garantía del cumplimiento de estos tratados y de las resoluciones emanadas de los organismos internacionales o supranacionales a los que hayan podido atribuirse competencias derivadas de la Carta Mag$\mathrm{na}^{7}$.

Este Informe se emite por el Consejo de Estado en respuesta a una consulta elevada por el Gobierno ${ }^{8}$ en noviembre de 2009 en la que se solicitaba un estudio sobre la garantía del cumplimiento del Derecho de la Unión Europea en España en el contexto de la problemática referida? . A la vista de las consultas planteadas el Consejo de Estado centra su Informe en la compleja tarea de explorar los diferentes mecanismos y procedimientos que permitan determinar la existencia de un incumplimiento de Derecho de la Unión por parte de una o varias Comunidades Autónomas u otros entes públicos y, en su caso, cómo repercutir sobre éstos las eventuales sanciones impuesta por las Instituciones de la Unión que pudieran recaer en el Estado con causa en tales incumplimientos ${ }^{10}$.

Al objeto de ofrecer al lector una panorámica de todo lo contenido en el Informe desgranaremos lo más significativo de los distintos capítulos del mismo en los siguientes epígrafes de este trabajo, haciendo especial hincapié en lo referido a las Comunidades Autónomas, por ser de mayor relevancia.

6 Arts. 259 y ss. del TFUE relativos al procedimiento por incumplimiento ante el Tribunal de Justicia de la UE que se sigue contra los Estados.

7 Un ejemplo práctico reciente de esta problemática puede consultarse en la noticia aparecida el diario Público en su edición de 19 de junio de 2011 «Bruselas denuncia a España por no depurar aguas residuales» en la que se da cuenta de una denuncia de Comisión Europea presentada contra el Gobierno Español que trae causa en el incumplimiento por parte de ciertas Comunidades Autónomas y municipios de la Directiva sobre depuración de aguas.

8 Art. 1.3 de la LO 3/1980 del Consejo de Estado. Vid. también sobre los informes del Consejo de Estado y su procedimiento de elaboración lo dispuesto en los Artículos 133 y 134 del RD 1674/1980, de 18 de julio, por el que se establecen el Reglamento Orgánico del Consejo de Estado.

9 El Gobierno formulaba fundamentalmente cuatro cuestiones. En primer lugar, se interesaba conocer cuáles son los supuestos en los que surge responsabilidad por incumplimiento y sus efectos; en segundo lugar, se consultaba sobre los procedimientos a los que es posible recurrir en el actual marco constitucional para mejorar la coordinación de las potestades normativas del Estado y de las Comunidades Autónomas, al objeto de garantizar el adecuado cumplimiento de las obligaciones de España en el desarrollo y ejecución del Derecho Europeo; en tercer lugar, se preguntaba sobre qué procedimientos permiten determinar la existencia de responsabilidad por incumplimiento de las Comunidades Autónomas u otros entes de la Administración; y, en cuarto lugar y último lugar, se solicitaba informe sobre aquellos mecanismos permiten hacer efectiva la responsabilidad de las Comunidades Autónomas o cualquier otro ente que haya incumplido con el Derecho Europeo.

10 Puede accederse al texto completo del Informe en la web oficial del Consejo de Estado. Téngase en cuenta que el Consejo de Estado ya ha elaboró en febrero de 2008 un Informe sobre la inserción del Derecho Europeo en el Ordenamiento español, en el que se tratan ciertas materias directamente relacionadas con las garantías del cumplimiento del Derecho Europeo. En esta misma web se puede acceder al texto completo del Informe de febrero de 2008. Vid. sobre este informe lo indicado por M. AZPITARTE SÁNCHEZ, «El Estado Autonómico unitario y el informe del Consejo de Estado sobre la inserción del Derecho europeo en el ordenamiento español» en Revista d'Estudis Autonòmics i Federals, no 9-Octubre de 2009, págs.129 y ss. Por otra parte, también aparece significativamente referida la cuestión de la garantía del cumplimiento del Derecho de la UE en el Informe de 16 de febrero de 2006, sobre modificaciones de la Constitución Española; en el Dictamen de 18 de marzo de 2009, sobre el anteproyecto de Ley sobre Libre Acceso y Ejercicio de las Actividades de Servicios, de 25 de junio de 2009; en el Dictamen de 25 de junio de 2009 sobre el anteproyecto de Ley por la que se reforme la Ley 7/1996, de 15 de enero, de Ordenación del Comercio Minorista. 


\section{SOBRE EL INCUMPLIMIENTO DEL DERECHO DE LA UNIÓN EUROPEA Y SU PROCEDIMIENTO DE SANCIÓN: IMPLICACIONES PARA LAS COMUNIDADES AUTÓNOMAS}

Comenzaremos señalando que el incumplimiento del Derecho de la Unión Europea por parte de un Estado miembro es un concepto dotado de contenido por la jurisprudencia del Tribunal de Justicia de la Unión Europea que, tal y como ha indicado la doctrina, se caracteriza por su significativa amplitud ${ }^{11}$. Así, un Estado miembro puede incurrir en incumplimiento, fundamentalmente, por tres motivos: por no realizar el debido desarrollo normativo de disposiciones de la $\mathrm{UE}^{12}$, por una inexistente o deficiente ejecución de la normativa comunitaria ${ }^{13}$ o por una falta de diligencia en la depuración de normas nacionales contrarias la legislación comunitaria ${ }^{14}$. El proceso por incumplimiento se regula en los artículos 258, 259 y 260 del TUE y consta de dos fases la precontenciosa y la contenciosa. Es preciso llamar la atención sobre el hecho de que, tal y como señala el informe del Consejo de Estado, la constatación fehaciente de la existencia de un incumplimiento del Derecho de la Unión Europea se produce desde el momento en que existe un pronunciamiento del Tribunal de Justicia al efecto ${ }^{15}$.

Por lo que al recurso por incumplimiento se refiere ${ }^{16}$, debe tenerse en cuenta que el Tratado de Lisboa ha introducido dos significativas novedades. La primera consiste en la eliminación del trámite del «dictamen motivado», que antes era obligatorio para la Comisión en caso de que quisiera formular denuncia ante el TJUE contra un Estado a fin de

11 G. ISAAC; Manual de Derecho Comunitario General, Barcelona, Ariel, 2000, págs. 389 y ss.

12 Los Tratados y la normativa comunitaria imponen a los Estados miembros el desarrollo normativo de ciertas disposiciones comunitarias; así sucede fundamentalmente con las Directivas de la Unión, que requieren en todo caso de la colaboración normativa de los Estados miembros para que éstos realicen su trasposición en plazo de forma completa y eficaz.

13 Los Estados miembros tienen la obligación, en el marco del principio de leal cooperación con la Unión, de ejecutar, cuando les corresponda, el Derecho de la UE.

14 Debe tenerse en cuenta que también constituye un incumplimiento del Derecho de la UE por parte de un Estado el mantenimiento dentro del ordenamiento nacional de aquellas normas que sean contrarias al Derecho de la Unión. En este caso, y en base al principio de supremacía de las normas de la UE reconocida en la Sentencia del TJCE Costa/Enel, toda antinomia entre una norma nacional y una norma europea se decidirá, en principio, a favor de la aplicación de la norma europea y la inaplicación de la norma nacional. No obstante, y aunque la norma nacional no se aplicara, el mantenimiento de la misma en el ordenamiento nacional puede constituir un incumplimiento. Vid. Declaración $\mathrm{n}^{\circ} 17$ aneja al Tratado de Lisboa relativa a la primacía. Vid. in extenso J.M. MARTÍNEZ SIERRA, La recepción constitucional del Derecho Comunitario, Valencia, Tirant lo Blanch, 2007.

15 Informe, pág. 212 y ss. El Consejo de Estado recoge en su informe el debate doctrinal existente a propósito de la determinación del momento en que el Estado Central debe poner en marcha medidas de reacción contra el posible incumplimiento del Derecho de la UE por parte de una Comunidad Autónoma o cualquier otro entre de la Administración.

$16 \mathrm{El}$ Consejo de Estado indica en su informe que, además del proceso por incumplimiento, es posible que se constate la inobservancia del Derecho de la UE por vía de una cuestión prejudicial; en tanto que cabe que el Tribunal de Justicia de la UE, al ser consultado por un órgano nacional respecto a la validez o eficacia de una norma de Derecho de la Unión, responda de modo que quepa interpretar la existencia de un incumplimiento. Sin embargo, considera el Consejo de Estado que el estudio de esta vía de declaración de incumplimiento es de menor relevancia para la problemática referida de la determinación y repercusión de la responsabilidad a las Comunidades Autónomas y otros entes en caso de que incurran en incumplimiento del Derecho de la UE. Informe, pág. 26. 
que éste procediera a ejecutar una previa sentencia por incumplimiento. La segunda novedad es la posibilidad de que, cuando la presunta inobservancia del Derecho de la Unión consista en el incumplimiento de la obligación de informar sobre las medidas de transposición realizadas respecto de una directiva, la Comisión, tras la realización de la fase precontenciosa, pueda acudir directamente ante el TJUE interesando la imposición de una sanción económica, sin necesidad de que tenga lugar un previo proceso declarativo del incumplimiento, como ocurre en el resto de $\operatorname{casos}^{17}$.

Por lo que al objeto de este trabajo interesa, es necesario destacar que aunque el Estado español alegue ante la Comisión o el Tribunal de Justicia de la Unión Europea que el incumplimiento por el cual ha sido encausado no es imputable a él sino a una Comunidad Autónoma o a cualquier otro ente de la Administración, esto no implicará en ningún caso que el Estado central deje de ser responsable ante la Unión de dicho incumplimiento y de la eventual sanción que se derive del mismo. Tales alegaciones no han sido consideradas por la Jurisprudencia del Tribunal Comunitario como eximentes en los procedimientos por incumplimiento contra el Estado. De esta forma han se han producido distintas Sentencias contra España a causa de incumplimientos del Derecho de la Unión de los que, sin embargo, eran efectivamente responsables una o varias Comunidades Autónomas, en tanto que titulares de la competencia en la materia referida al ámbito donde se había producido el incumplimiento ${ }^{18}$. Tampoco van a ser tenidas en cuenta alegaciones del Estado que, referidas a la Comunidades Autónomas o u otros entes, se fundamenten en argumentos políticos, como la disolución de las Cámaras autonómicas; argumentos jurídicos, como que existe un error de compresión de la normativa o que existe incumplimiento por parte de otros Estados y sus regiones; argumentos económicos, como ausencia de recursos presupuestarios suficientes; argumentos de política social, como una situación local conflictiva o la resistencia de la población; argumentos técnicos, como problemas tecnológicos o de infraestructura; o argumentos relativos al propio personal de la administración autonómica, como falta de formación o carencia de funcionarios. De esta forma, y aún cuando la responsabilidad efectiva de un incumplimiento del Derecho de la Unión Europea traiga causa en la acción u omisión de una Comunidad Autónoma, o de cualquier otro ente de la Administración, la responsabilidad por dicho incumplimiento siempre recaerá sobre el Estado. De modo que la implicación para la Comunidad Autónoma o el ente de la Administración será, a los efectos de la posible sanción impuesta por Sentencia del TJUE, en principio inexistente.

17 Art. 260.3 del TFUE. Vid. sobre el cálculo de la sanción económica Comunicación de la Comisión Aplicación del artículo 260, apartado 3, del TFUE, publicada en DOUE C12 de 15 de enero de 2011.

18 Vid. por ejemplo, en este sentido el Punto 37 de la STJCE de 13 de septiembre de 2001 (Asunto C417/99) en el que se indica «cualesquiera que sean las normas de organización o de competencia vigentes en el territorio español, el Reino de España está obligado, conforme a lo dispuesto por el artículo 189 del Tratado CE (actualmente artículo 249 (E), a velar por la aplicación exacta y completa de la Directiva. En consecuencia, las explicaciones de dicho Estado, según las cuales compete, de manera exclusiva, a las Comunidades Autónomas velar por la adaptación del Derecho interno al artículo 3 de la Directiva, no le pueden eximir de sus obligaciones respecto a las disposiciones del artículo 169 del Tratado.» Otras Sentencias contra España que traen causa en incumplimientos autonómicos son: la STJCE de 1 de Octubre de 1998 (Asunto C71/97), STJCE de 2 de julio de 2002 (Asunto C499/99), STJCE de 9 de diciembre de 2004 (Asunto C79/103), STJCE de 9 de junio de 2005 (Asunto C287/02), y STJCE de 9 de marzo de 2006 (Asunto C323/03). 
Otra cuestión distinta es cómo el Estado puede, en el orden interno, establecer mecanismos preventivos para que el incumplimiento no llegue a producirse o, en caso de ocurrir, se puedan repercutir las consecuencias del mismo sobre el ente incumplidor. Así, aunque las Comunidades Autónomas responsables efectivas del presunto incumplimiento no serán directamente parte del procedimiento ante el Tribunal de Justicia — pues solamente lo será el Reino de España—, sí se han articulado en el orden interno español diversos instrumentos para posibilitar que las Comunidades Autónomas y Estado colaboren en el procedimiento ante el $\mathrm{TJUE}^{19}$. En primer lugar, existe un deber de información por parte del Estado hacia la Comunidad Autónoma presuntamente responsable del incumplimiento, correspondiendo al Ministro de Asuntos Exteriores, por vía de la Secretaría de Estado de Política Exterior y para la Unión Europea, comunicar a la Comunidad o Comunidades Autónomas aquellos escritos de queja, cartas de emplazamiento, dictámenes motivados y demás comunicaciones recibidas de la Comisión relativas a un presunto incumplimiento que afecten a sus competencias. En segundo lugar, es responsabilidad del Estado central, y en concreto en la Secretaría de Estado de Política Exterior y para la Unión Europea, la coordinación de las diferentes Administraciones autonómicas afectadas por el presunto incumplimiento a los efectos de responder a las acusaciones formuladas ante el Tribunal. Para ello la Comunidad Autónoma responsable del presunto incumplimiento transmitirá a la Secretaría de Estado aquellas alegaciones y datos que estime pertinentes para la elaboración de la respuesta. Además, si se considera oportuno podrán celebrarse reuniones a los efectos de preparar la respuesta a las acusaciones; algo que a juicio del Consejo de Estado debería ser de naturaleza obligatoria y no potestativa, como es actualmente ${ }^{20}$. Y en tercer lugar, en los supuestos en los que finalmente se interponga recurso ante el Tribunal de Justicia contra España — cuando el posible presunto tenga su origen en una acción y omisión de una Comunidad Autónoma- ésta podrá designar Abogados o asesores para que asistan, en su caso, al Abogado que represente al Estado en el procedimiento por incumplimiento.

\section{LA ACTUAL SITUACIÓN DEL ORDENAMIENTO JURÍDICO ESPAÑOL ANTE EL INCUMPLIMIENTO DEL DERECHO DE LA UNIÓN EUROPEA POR PARTE DE UNA COMUNIDAD AUTÓNOMA: INSTRUMENTOS GENERALES DE PREVENCIÓN Y REACCIÓN}

El Consejo de Estado dedica gran parte de su informe a ordenar y sistematizar los diferentes mecanismos contemplados actualmente en el ordenamiento jurídico español que permiten tanto la prevención de posibles incumplimientos del Derecho de la UE por parte de las Comunidades Autónomas, como la eventual repercusión sobre ellas de las po-

19 Resolución de 24 de marzo de 1998, de la Subsecretaría, por la que se dispone la publicación del Acuerdo de 11 de diciembre de 1997 de la Conferencia para Asuntos Relacionados con las Comunidades Europeas, relativo a la participación de las Comunidades Autónomas en los procedimientos ante el Tribunal de Justicia de las Comunidades Europeas, publicada en el BOE n 79 de 2 de abril de 1998.

20 Informe, pág. 222. 
sibles sanciones impuestas al Estado por parte del Tribunal de Justicia. Los mecanismos que según el Consejo de Estado pueden servir como instrumentos generales de prevención y reacción frente al incumplimiento del Derecho de la UE causados por acciones u omisiones de las Comunidades Autónomas son, fundamentalmente, siete. En primer lugar, la competencia estatal en materia de relaciones internacionales contenida en la regla tercera del apartado 1 del artículo 149 CE y su eventual conexión con el artículo 93 CE; en segundo lugar, la utilización de títulos competenciales del Estado de carácter básico o transversal; en tercer lugar, la cláusula general de supletoriedad del Derecho del Estado prevista en el art. 149.3 CE; en cuarto lugar, las leyes de armonización del art. 150.3 CE; en quinto lugar, los poderes especiales reconocidos al Estado en virtud del artículo 155 CE; en sexto lugar, los procedimientos ante el Tribunal Constitucional, a los que puede recurrir el Estado en contra la de las Comunidades Autónomas; y, en séptimo lugar, los procedimientos ante la Jurisdicción Contencioso Administrativa.

\section{III.1. LA REGLA TERCERA DEL APARTADO 1 DEL ARTíCULO 149 CE Y SU CONEXIÓN CON EL ARTÍ́CULO 93 CE}

Por lo que respecta a la regla tercera del apartado 1 del artículo 149 de la Constitución y su eventual conexión con el artículo 93, el Consejo de Estado recuerda que, tal y como ha indicado el Tribunal Constitucional en su jurisprudencia, no toda conexión de una determinada materia con el ámbito internacional o comunitario otorga naturaleza de «relación internacional» a dicha materia. De esta forma se limita la vis expansiva que pudiera tener para el Estado su competencia exclusiva en materia internacional, ${ }^{21}$ que queda reducida a su núcleo más esencial ${ }^{22}$. Así, la pertenencia de España a la Unión Europea no justifica para que sobre el título competencial del art. 149.1.3 $3^{\mathrm{a}}$ todos los asuntos de la Unión pasen a ser competencia exclusiva del Estado central. Sin embargo, dicha regla tercera en conexión con el art. 93 CE sí que atribuye al Estado la competencia respecto de la función de garantía de la responsabilidad internacional derivada de los Tratados. Aunque, tal y como ha indicado el Tribunal Constitucional, esta competencia sobre la responsabilidad internacional no puede constituirse en un título competencial específico a favor del Estado que legitime la alteración de la distribución competencial operada por la Constitución entre el nivel central y el autonómico. No obstante lo anterior, el Estado sí debiera tener a su disposición los instrumentos necesarios para poder realizar su actividad como garante de la responsabilidad internacional de forma efectiva ${ }^{23}$.

21 FJ 3 de la STC 80/1993: «La dimensión externa de un asunto no puede servir para realizar una interpretación expansiva del art. 149.1.3 C.E., que venga a subsumir en la competencia estatal toda medida dotada de una cierta incidencia exterior, por remota que sea, ya que si asi fuera se produciría una reordenación del propio orden constitucional de distribución de competencias entre el Estado y las Comunidades Autónomas».

22 FJ 5 de la STC 165/1994; según el TC se circunscribe fundamentalmente a tres facultades: la celebración de Tratados, la representación diplomática exterior y la creación de obligaciones internacionales para España y la responsabilidad de cumplimiento respecto de las mismas.

23 Debe tenerse en cuenta que no sólo la CE señala al Estado como garante de las obligaciones del Derecho de la UE, sino que está función también viene determinada por el propio Derecho de la UE, vid. supra introducción. 
Por tanto, tal y como señala el Consejo de Estado en su informe, las facultades de actuación del Estado en su condición de garante del cumplimiento del Derecho de la Unión si bien no pueden amparar la usurpación de competencias autonómicas, sí que atribuyen al Estado central ciertas facultades en la materia para que dicha función pueda ser efectivamente realizada; tales como el poder de vigilancia y control, la obligación de advertir a la Comunidad Autónoma de los incumplimientos que pueda estar llevando a cabo, el establecimiento de sistemas de cooperación y coordinación o, incluso, sistemas de compensación interadministrativos de la eventual responsabilidad financiera derivada del incumplimiento ${ }^{24}$. Finalmente, y dentro de esta función de garante amparada por el art. 93, también se indica por el Consejo de Estado que la jurisprudencia del Tribunal Constitucional reconoce implícitamente y en cierta medida la posibilidad de que el Estado central pudiera llegar a actuar en sustitución de una o varias Comunidades Autónoma una vez que, por omisión de sus obligaciones derivadas del Derecho de la UE, hubieran dado lugar a un efectivo incumplimiento ${ }^{25}$. Este eventual desplazamiento o sustitución de la Comunidad Autónoma podría ser admisible en tanto que se hubiera constatado efectivamente el incumplimiento, sin que quepa en ningún caso una sustitución «anticipada» al mismo. De este modo el título competencial del art. 93 en relación con la regla tercera del 149.1 podría «entrar en juego» para permitir al Estado atribuirse la capacidad de actuar en ámbitos competenciales autonómicos, en su condición de garante de la responsabilidad de cumplimiento del Derecho de la Unión Europea, cuando dicho incumplimiento haya sido efectivamente verificado y tenga como responsable efectivo a una Comunidad Autónoma ${ }^{26}$.

\section{III.2. LA UTILIZACIÓN DE TÍTULOS COMPETENCIALES ESTATALES DE NATURALEZA BÁSICA Y/O TRANSVERSAL}

Partiendo de la base del principio de que la adhesión de España a la Unión Europea no implica la alteración del reparto competencial existente entre Estado y Comunidades Autónomas, el Consejo de Estado examina las posibilidades de que el Estado central - a fin de garantizar el cumplimiento del Derecho de la Unión Europea por parte de las Comunidades - pueda recurrir a dictar normativa estatal de carácter básico o transversal en ciertas materias que satisfaga las obligaciones derivadas del Derecho de la Unión; evitando así que una posible inactividad de las Comunidades Autónomas condujera a una sanción por incumplimiento, fundamentalmente en lo relativo a la transposición de directivas. La Constitución reconoce al Estado en determinados ámbitos la competencia exclusiva para dictar normativa básica, como la relativa a las bases y coordinación de la planificación económica, el sistema monetario, la sanidad, o el medio ambiente. Señala el Consejo de Estado que no es infrecuente que el Estado apruebe normas básicas en dichos ámbitos que per-

24 Informe, págs. 85 a 87.

25 FJ 3 de la STC 80/1993: «En cualquier caso, los poderes de control y vigilancia que, para el aseguramiento de la ejecución, deban corresponder al Estado lo que no pueden es afectar a la competencia misma que constitucionalmente deban desarrollar y ejercitar las Comunidades Autónomas, desplazándola o sustituyéndola anticipadamente por el ejercicio de poderes que, en ese caso, no serán ya de control y vigilancia.»

26 Informe, pág. 89. 
mitan tener por transpuesta una directiva comunitaria, sin perjuicio de que ésta normativa básica estatal sea ulteriormente desarrollada y ejecutada por las Comunidades Autónomas. Dicha actividad normativa básica no ha conllevado una significativa conflictividad entre el Estado y las Comunidades, aunque sí se han producido ciertos enfrentamientos entre ambos niveles a propósito de la ejecución. Y, por lo que a la garantía del cumplimiento del Derecho de la Unión Europea se refiere, éste es un sistema que permite asegurar la observancia de las obligaciones derivadas del Derecho de la Unión Europea a los ojos de las instituciones de la Unión Europea; pues contribuye a solucionar la problemática comprobación de que efectivamente una directiva comunitaria ha sido transpuesta cuando son varios los niveles y administraciones llamados a su transposición.

\section{III.3. La ClÁusula general de Supletoriedad Del Derecho del Estado PREVISTA EN EL ART. 149.3 CE}

El Consejo de Estado examina en su informe la cláusula de supletoriedad del Derecho del Estado contenida en el artículo 149.3 CE como uno de posibles instrumentos de prevención y reacción frente un incumplimiento de Derecho de la Unión Europea de naturaleza normativa por parte de una Comunidad Autónoma ${ }^{27}$. Tal y como ha sido indicado por la Doctrina desde hace ya varios lustros, el recurso a normativa de carácter supletorio podría evitar una sanción por incumplimiento que tuviera su origen en la inactividad normativa de una o varias Comunidades Autónomas; constituyéndose así en el instrumento que mejor conjuga la responsabilidad exterior y la autonomía interior ya que permite garantizar el cumplimiento $y$, a la vez, no rompe el reparto interno de competencias ${ }^{28}$.

\section{III.4. LAS LEYES DE ARMONIZACIÓN DEL ART. 150.3 CE}

Las leyes de armonización del art. 150.3 CE son otra de las vías, que a juicio del Consejo de Estado, pueden servir como vía de garantía del cumplimiento del Derecho de la Unión Europea por las Comunidades Autónomas. Sin embargo, lo que más destaca en el informe son las debilidades que este instrumento ofrece para el fin propuesto. En pri-

27 El informe recoge la discusión existente al respecto del uso de este instrumento como mecanismo para garantizar el cumplimiento del Derecho de la UE. Por un lado, hay quien defiende que, sobre la base de este título competencial, el Estado podría dictar en todo caso legislación de carácter supletorio en virtud de la cual se diera cumplimiento a las obligaciones de desarrollo normativo derivadas del Derecho de la Unión. Otros, por su parte, defienden que sólo cabría recurrir al este mecanismo cuando se constante efectivamente el incumplimiento por parte de una Comunidad Autónoma. Finalmente, existe otra posición que niega cualquier tipo de operatividad práctica al uso de la cláusula de supletoriedad como mecanismo de garantía del cumplimiento del Derecho de la Unión por parte de las Comunidades Autónomas. Vid. in extenso sobre el debate relativo a la aplicación supletoria del derecho estatal como garantía de la ejecución de obligaciones normativas europeas en el ámbito autonómico en J.F. SÁNCHEZ BARRILAO, «La supletoriedad como garantía en la ejecución del Derecho Europeo por las Comunidades Autónomas» en Revista de Derecho Constitucional Europeo, no 1, de Junio de 2004, págs. 256 y ss.; Vid. también Informe del Consejo de Estado de 15 de diciembre de 2010, pág. 89.

28 P. PÉREZ TREMPS, Comunidades Autónomas, Estado y Comunidad Europea, Madrid, Ministerio de Justicia, 1987, págs. 193 a 196. 
mer lugar, sólo cabe recurrir a este tipo de normas con un carácter subsidiario y en todo caso, según el Consejo, deberían articularse procedimientos de audiencia previos entre el Estado y las Comunidades Autónomas cuando se pretenda usar este mecanismo de garantía. También se señala en el informe, como una significativa debilidad, que este tipo de normas sólo puede ser utilizada para «armonizar principios» y en ningún caso para establecer una regulación detallada como la que puede derivarse de las obligaciones de desarrollo normativo que implica el Derecho de la Unión Europea. Todas estas cuestiones hacen que el Consejo de Estado solo reconozca a las leyes de armonización una virtualidad muy relativa como instrumentos de garantía del Derecho de la Unión, a las que solo cabría recurrir en caso de no existir posibilidad de acudir a otros mecanismos ${ }^{29}$.

\section{III.5. SOBRE LA UTILIZACIÓN DEL ARTÍCULO 155 CE}

Tal y como ha reconocido la jurisprudencia del Tribunal Constitucional el artículo de $155 \mathrm{CE}$ es un mecanismo al que solo es posible acudir en casos de especial y significativa gravedad de dimensión excepcional ${ }^{30}$. Sobre la base de este uso excepcional, el Consejo de Estado estima que únicamente sería posible recurrir al artículo $155 \mathrm{CE}$ como ultima ratio cuando, debido a un incumplimiento de Derecho de la UE provocado por la acción u omisión de una Comunidad Autónoma, recayera sobre el Estado una significativa y excepcionalmente gravosa sanción económica, y que por lo tanto atentara gravemente al interés general. El Consejo de Estado indica en su informe que el procedimiento previsto el artículo $155 \mathrm{CE}$, aunque excepcional, sí puede ser un instrumento eficaz en manos del Estado frente un incumplimiento de Derecho de la Unión por parte de una Comunidad Autónoma. Afirma el informe que, además de servir para remediar incumplimientos de naturaleza normativa y ejecutiva, permitiría incluso repercutir, en su caso, sobre la Comunidad Autónoma efectivamente responsable del incumplimiento del Derecho de la Unión la eventual sanción económica que el Tribunal de Justicia impusiera a España por dicho incumplimiento ${ }^{31}$. No obstante, sí debemos reseñar aquí que, contrariamente a la opinión del Consejo de Estado manifestada en su informe, existen distintos autores que consideran dudosa su operatividad de cara a su utilización como instrumento de garantía de cumplimiento del Derecho de la Unión Europea ${ }^{32}$.

\section{III.6. LOS PROCEDIMIENTOS ANTE EL TRIBUNAL CONSTITUCIONAL}

El Consejo de Estado considera que los procedimientos que el Estado puede instar contra las Comunidades Autónomas ante el Tribunal Constitucional no constituyen una vía realmente operativa para prevenir o, en su caso, repercutir las infracciones del Derecho de la UE realizadas por las Comunidades Autónomas. En primer lugar, destaca el Informe

29 Informe, págs. 117 a 123.

30 STC 6/1982.

31 Informe, págs. 124 a 130.

32 P. PÉREZ TREMPS, op. cit.; pág. 213. 
que los plazos previstos para la interposición de los recursos ante el Tribunal Constitucional son muy limitados; además, este Tribunal, ya de por sí recargado de trabajo, no es generalmente lo suficientemente rápido en su toma de decisiones como para poder evitar mediante su actividad la posible sanción que impuesta por el Tribunal de Justicia de la Unión Europea pudiera recaer sobre el Estado. Por otra parte, también puede producirse cierta contradicción en la posición del Estado pues, en el nivel europeo estaría litigando ante la jurisdicción comunitaria en contra de la declaración del supuesto incumplimiento, mientras que en el nivel nacional estaría actuando como demandante contra una Comunidad Autónoma que fuera la responsable efectiva del incumplimiento por el cual el Estado está encausado ante el TJUE. El Consejo de Estado únicamente sugiere que los conflictos de competencias, y concretamente, los de naturaleza negativa, podrían constituir una vía en cierta medida idónea para poner fin a omisiones autonómicas de carácter no normativo que implicarán un incumplimiento del Derecho de la Unión.

\section{III.7. PROCEDIMIENTOS ANTE LA JURISDICCIÓN CONTENCIOSO ADMINISTRATIVA}

Finalmente, el Consejo de Estado examina la posibilidad de acudir a los procedimientos ante la jurisdicción contencioso administrativa. Considera el informe que, aunque eventualmente el Tribunal Supremo — en virtud de los principios de efecto directo y primacía del Derecho de la Unión y de la competencia de control de la potestad reglamentaria - ha no sólo inaplicado sino también anulado normas reglamentarias contrarias del Derecho de la Unión Europea, la virtualidad de los recursos contenciosos administrativos para poner fin al incumplimiento del Derecho de la Unión Europea por parte de una Comunidad es muy relativa. Entre otras causas, por ejemplo, por lo breve de los plazos para la interposición de recursos, que por norma general es sólo de dos meses. Por otra parte, el Consejo de Estado también remarca la ausencia en nuestro ordenamiento de vías administrativas ágiles que permitan al Estado central reaccionar frente a incumplimientos de Derecho de la Unión Europea por parte de las Comunidades Autónomas; y sugiere al respecto la posibilidad que por ley se regule un procedimiento administrativo que permita eliminar de forma ágil y rápida normas reglamentarias, tanto del Estado como de las Comunidades Autónomas, que hayan sido previamente declaradas incompatibles con el Derecho del Unión Europea por la Comisión o por el Tribunal de Justicia ${ }^{33}$.

\section{SOBRE LOS MECANISMOS Y PROCEDIMIENTOS DE REPERCUSIÓN DE LA RESPONSABILIDAD POR INCUMPLIMIENTO DEL DERECHO DE LA UNIÓN A LAS COMUNIDADES AUTÓNOMAS}

Vistos los distintos mecanismos de prevención y reacción, previstos por el ordenamiento jurídico nacional, ante el incumplimiento del Derecho de la UE por parte de las

33 Informe, págs. 144-152. 
Comunidades Autónomas; el Consejo de Estado analiza en su informe las distintas vías previstas para posibilitar la repercusión a la Comunidad efectivamente culpable de la infracción, de una eventual sanción que recaiga sobre el Estado, impuesta por las Instituciones Europeas con causa en dicho incumplimiento.

La jurisprudencia del Tribunal Constitucional ha admitido que la existencia de una responsabilidad internacional ab extra derivada del incumplimiento de obligaciones contraídas por España —a la que tiene que hacer frente el Estado, en tanto que garante de la responsabilidad internacional - no impide que ésta sea posteriormente repercutida ab intra sobre el ente causante de la infracción, y específicamente sobre las Comunidades. No obstante, como se señala por el Consejo de Estado, la habilitación para que el Estado repercuta ab intra la eventual responsabilidad a la que hubiera tenido que hacer frente ab extra no debería suponer en ningún caso la alteración del reparto competencial existente entre Estado y Comunidades Autónomas. Aunque sí se reconoce que al Estado debería corresponderle la competencia sobre la coordinación y los sistemas de compensación interadministrativa de la responsabilidad ab intra a la que, en su caso, hubiera lugar $^{34}$. Actualmente existen dos tipos de procedimientos de repercusión de las sanciones derivadas de incumplimiento del Derecho de la Unión sobre las Comunidades Autónomas, por un lado, un régimen general y, por otro, cuatro regulaciones sectoriales que han sido articuladas normativamente ad casum.

\section{IV.1. RÉGIMEN GENERAL DE REPERCUSiÓN DE LA RESPONSABILIDAD: LA Disposición Adicional Primera de la Ley de ECONOMía Sostenible}

El informe del Consejo de Estado dedica varias de sus páginas al examen de las previsiones del proyecto de Ley de Economía Sostenible relativas a la repercusión de la responsabilidad derivada del incumplimiento del Derecho de la Unión Europea a los entes de la Administración pública causantes del mismo. Dicho proyecto de Ley, cuyo anteproyecto fuera informado por el Consejo de Estado ${ }^{35}$, ha entrado definitivamente el vigor el pasado 6 de marzo de 201136; conteniéndose finalmente en su Disposición Adicional primera un procedimiento que regula el régimen general relativo a la repercusión ab intra sobre los entes de cualquiera de las Administraciones Públicas que fueran los responsables de los incumplimientos de Derecho de la UE que hubieran dado lugar a sanciones $^{37}$.

34 FJ 5 de la STC 79/1992: «Pero esa responsabilidad «ad extra» de la Administración del Estado no justifica la asunción de una competencia que no le corresponde, aunque tampoco le impide repercutir ad intra, sobre las Administraciones públicas autonómicas competentes, la responsabilidad que en cada caso proceda.»

35 Vid. Consideración Décima del Dictamen 215/2010, de 18 de marzo de 2010, sobre el anteproyecto de Ley de Economía Sostenible. En dicha consideración se realiza un extenso y profuso análisis del mecanismo general de repercusión ab intra de la responsabilidad por incumplimiento del Derecho de la UE; señalándose por parte del Consejo de Estado las debilidades y fortalezas del mismo.

36 Ley 2/2011, de 4 de marzo, de Economía Sostenible.

37 DA $1^{a}$ de la Ley 2/2011, de 4 de marzo, de Economía Sostenible: «1. Las Administraciones Públicas y cualesquiera otras entidades integrantes del sector público que, en el ejercicio de sus competencias, incumplieran obligaciones derivadas de normas del derecho de la Unión Europea, dando lugar a que el Reino de España sea sancionado por las insti- 
Este procedimiento, cuyo reglamento de desarrollo corresponde elaborar al Gobierno, permite que el Consejo de Ministros repercuta en el ente de la Administración origen del incumplimiento, previa audiencia al mismo, la parte que le sea imputable de la sanción que fuera impuesta al Estado central en virtud de dicho incumplimiento. El Consejo de Estado se muestra favorable a este procedimiento; sin embargo, a su juicio, aún cabría introducir en él algunas mejoras, tales como un mayor detalle en la regulación, o una mayor determinación y concreción en la reglas de imputación de la responsabilidad, extremos estos que precisa en las recomendaciones finales de su Informe ${ }^{38}$.

\section{IV.2. REGÍMENES SECTORIALES DE REPERCUSIÓN DE LA RESPONSABILIDAD}

Además del nuevo régimen general de repercusión de la responsabilidad, contenido en la Ley de Economía Sostenible, el Consejo de Estado repasa en su informe cuatro regímenes sectoriales de repercusión de la responsabilidad que están específicamente regulados los ámbitos de la gestión de fondos europeos ${ }^{39}$, el déficit excesivo ${ }^{40}$, calidad de aguas $^{41}$ e incorporación de la directiva de servicios ${ }^{42}$.

En estos cuatro regímenes sectoriales, el Consejo de Estado aprecia la existencia de ciertas deficiencias comunes, por un lado, en cuanto a la determinación de los criterios de imputabilidad de la responsabilidad del ente cuyo incumplimiento da lugar a la sanción al Estado, por otro lado, en la ausencia de una regulación del procedimiento de repercusión de la responsabilidad, del que solo se indica que contendrá un trámite de audiencia al ente de la Administración origen del incumplimiento. En conclusión, el Informe señala que estos regímenes sectoriales están constituidos únicamente por una

tuciones europeas asumirán, en la parte que les sea imputable, las responsabilidades que se devenguen de tal incumplimiento, de conformidad con lo previsto en esta disposición y en las de carácter reglamentario que, en desarrollo y ejecución de la misma, se dicten. 2. El Consejo de Ministros, previa audiencia de las Administraciones o entidades afectadas, será el órgano competente para declarar la responsabilidad por dicho incumplimiento y acordar, en su caso, la compensación de dicha deuda con las cantidades que deba transferir el Estado a la Administración o entidad responsable por cualquier concepto, presupuestario y no presupuestario. En dicha resolución que se adopte se tendrán en cuenta los hechos y fundamentos contenidos en la resolución de las instituciones europeas, se recogerán los criterios de imputación tenidos en cuenta para declarar la responsabilidad, y se acordará la extinción total o parcial de la deuda. Dicho acuerdo se publicará en el Boletín Oficial del Estado. 3. Se habilita al Gobierno para desarrollar reglamentariamente lo establecido en la presente disposición, regulando las especialidades que resulten aplicables a las diferentes Administraciones Públicas y entidades a que se refiere el apartado 1 de esta disposición.»

38 Informe, págs. 172 y 173 . Téngase en cuenta también lo indicado en la Consideración Décima del Dictamen 215/2010, de 18 de marzo de 2010, sobre el anteproyecto de Ley de Economía Sostenible.

39 Artículo 44 de la Ley 55/1999, de 29 de diciembre de medidas fiscales, administrativas y del orden social - Responsabilidad financiera derivada de la gestión de los fondos procedentes de la UE.

40 Art. 10.5 del Real Decreto Legislativo 2/2007, de 28 de diciembre, por el que se aprueba el texto refundido de la Ley General de estabilidad presupuestaria.

41 Artículo 121 bis del Real Decreto Legislativo 1/2001, de 20 de julio, por el que se aprueba el texto refundido de la Ley de Aguas. - Responsabilidad comunitaria. Téngase en cuenta que este artículo y el mecanismo de repercusión que prevé tienen su origen, según indica el Consejo de Estado en la condena recaída para España en la Sentencia del TJUE de 25 de noviembre de 2003 (Asunto C 278/01).

42 Disposición final cuarta de la Ley 17/2009, de 23 de noviembre, sobre el libre acceso a las actividades y servicios. - Compensación de deudas en caso de responsabilidad por incumplimiento.» 
cláusula legalmente prevista relativa a la posibilidad de repercusión de la responsabilidad, y que carecen de unos criterios claros para delimitar la responsabilidad que en su caso corresponda repercutir, y también de un procedimiento efectivamente reglado que permita la efectiva repercusión de la sanción al ente de la administración incumplidor ${ }^{43}$.

\section{EXPERIENCIAS EN DERECHO CONSTITUCIONAL COMPARADO}

Dentro del Informe se ha incluido un interesante estudio de Derecho comparado dedicado al análisis de la normativa existente en otros Estados miembros de la Unión Europea relativa a los procedimientos que permiten prevenir, reaccionar $y$, en su caso, repercutir la sanción derivada del incumplimiento del Derecho de la Unión sobre el ente infraestatal o de la Administración que fuera el responsable efectivo de dicho incumplimiento. En concreto en el informe se estudian los referentes de Italia, Alemania, Bélgica y Austria; todos ellos caracterizados por tratarse de Estados descentralizados que cuentan con regulación constitucional al respecto del incumplimiento de Derecho de la Unión $^{44}$. Tal y como indica el Consejo de Estado, los cuatro sistemas analizados se sirven fundamentalmente de dos procedimientos ante el incumplimiento, por un lado, la posibilidad de que el Estado pueda sustituir a los entes infraestatales y, por otro lado, la facultad reconocida al Estado para repercutir la eventual sanción sobre el ente infraestatal responsable efectivo del incumplimiento ${ }^{45}$.

\section{V.1. ITALIA}

En el caso italiano, la Constitución prevé desde 2001 en su artículo 120 la posibilidad de que el Gobierno de la República pueda actuar en lugar de los órganos de las regiones, de las ciudades metropolitanas, provincias y municipios en caso de que éstos incurran en incumplimiento del Derecho de la Unión Europea ${ }^{46}$. El desarrollo legislativo de esta previsión constitucional se realiza a través de la Ley ${ }^{\circ} 11$ de 2005, conocida como «Legge Buttiglione ${ }^{47}$. El modelo italiano se caracteriza, por reconocer al Estado central la facultad ge-

43 Informe, págs. 169-171.

44 Téngase en cuenta que el Reglamento Orgánico del Consejo de Estado establece la posibilidad de tener en cuenta la experiencias en Derecho comparado sobre la materia que sea objeto de Informe. Sobre la significación e importancia del Derecho constitucional Comparado en el momento actual vid. M. SALVADOR MARTÍNEZ, «Derecho Constitucional comparado en el contexto de la integración supranacional y globalización» en Teoría y Realidad Constitucional, nº. 21, 2008, págs. 375 a 395.

45 Informe, págs. 205-207.

46 La redacción actual de este artículo fue introducida por la Ley Constitucional de 18 de octubre de 2001, n 3, por la que se modifica el Título V de la Segunda Parte de la Constitución, publicada en la Gazzetta Ufficiale $\mathrm{n}^{\circ} 248$ de 24 de octubre de 2001.

47 Ley de 4 de febrero de $2005, n^{\circ} 11$, de normas generales sobre la participación de Italia en el proceso normativo de la UE y sobre los procedimientos de ejecución de las obligaciones comunitarias, publicada en la Gazzetta Ufficiale $\mathrm{n}^{\circ} 37$ de 15 de febrero de 2005. Tanto el art. 120 de la Constitución como la Ley Buttiglione tienen su antecedente en la Legge «La Pergola» de 1989 en la que comenzó a regular la responsabilidad por incumplimiento del Derecho de la UE por parte de los entes infraestatales italianos. 
neral de sustituir al ente infraestatal que sea origen del incumplimiento, por causa de su inactividad o ante el eventual grave peligro de su actuación-, siempre y cuando éste conlleve significativas repercusiones para Italia. Se habla así de una competencia estatal de «segunda instancia» respecto de las competencias «en primera instancia» de los entes descentralizados, que se ve preterida por la actuación estatal en caso de incumplimiento. No obstante, la actuación del Estado va a tener un carácter claudicante, en tanto que está perderá sus efectos cuando se produzca la correcta actuación del ente descentralizado con la que se ponga fin al incumplimiento. Lo más característico del modelo italiano lo constituye la denominada «legge comunitaria». Se trata de una Ley del Parlamento italiano en la que, al objeto de cumplir con las obligaciones derivadas del Derecho de la UE, se verifica el estado de conformidad del ordenamiento interno italiano con el ordenamiento comunitario, pudiéndose incorporar además mediante ella al ordenamiento aquellos contenidos del Derecho de la Unión que fuera necesario, o facultar a través suyo al Ejecutivo para que realice dicha incorporación ${ }^{48}$. Finalmente, el modelo italiano se cierra con las previsiones contenidas en la Legge finanzaria 2007, en la que se establece la posibilidad de que el Estado central repercuta sobre los entes descentralizados la sanción que eventualmente hubiera podido imponerse a Italia derivada del incumplimiento ${ }^{49}$.

\section{V.2. Alemania}

La Ley Fundamental de Bonn incluye en su artículo 104a las bases del reparto de las cargas derivadas del incumplimiento del Derecho de la Unión Europea entre la Federación y los Länder. Lo previsto en el art. 104a tiene su desarrollo normativo en la Ley, de 5 de septiembre de 2006, sobre el reparto de cargas entre la Federación y los Länder por el incumplimiento de las obligaciones internacionales y supranacionales ${ }^{50}$. El sistema germano pivota sobre el principio general de que las eventuales cargas derivadas del incumplimiento del Derecho de la Unión se repartirán en función de la distribución interna de competencias y del grado de responsabilidad que la Federación y/o los Länder en el incumplimiento origen de la sanción. Se prevé, además, que la Federación pueda solicitar de los Länder responsables el reembolso de la parte que les corresponda de la sanción económica recaída sobre la República Federal Alemana, a la que la ésta hubiera hecho frente.

A este sistema general se suma lo previsto específicamente en el apartado 5 del artículo 109 de la Ley Fundamental para el cumplimiento de la disciplina presupuestaria impuesta por el pacto de estabilidad y crecimiento contenido en el art. 126 del TFUE ${ }^{51}$. En caso de sanción impuesta a Alemania por incumplimiento de esta previsión contenida en

48 Vid. por ejemplo la «Ley Comunitaria 2009, Ley 4 de junio de 2010, nº 96, publicada en la Gazzetta Ufficiale $\mathrm{n}^{\circ} 146$ de 25 de junio de 2010.

49 Artículo 1. Apartados 1214 y siguientes de la Ley Financiera 2007, publicada en la Gazzetta Ufficiale $n^{\circ} 299$ de 27 de diciembre de 2006; en los que se detalla el procedimiento de repercusión.

50 Conocida también como «Lastentragungsgesetz», publicada en la Bundesgesetzblatt I, 2098 y 2105.

51 Vid. in extenso sobre el art. 109.5 de la Ley Fundamental y la responsabilidad financiera por incumplimiento del pacto de estabilidad en el Alemania en J. ALGUACIL GÓNZALEZ-AURIOLES, «El federalismo alemán ante integración Europea en Revista de Derecho Constitucional Europeo n 6, Julio-Diciembre 2006, págs 216 y ss. 
el Derecho originario de la Unión, se establece que, con carácter general, la Federación asumirá el sesenta y cinco por ciento de la sanción, mientras que el treinta y cinco restante corresponderá a los Länder ${ }^{52}$.

\section{V.3. Austria}

En Austria también existe desde 1994 una previsión constitucional referida a la garantía de cumplimiento del Derecho de la Unión Europea. Se prevé así que si un Land, en el marco de sus competencias, no cumpliera en tiempo y forma con las obligaciones derivadas del Derecho de la UE que le corresponda — siempre y cuando dicho incumplimiento haya sido declarado en virtud de resolución de los órganos jurisdiccionales de la Unión — la Federación podrá asumir la competencia para adoptar las medidas oportunas para poner remedio a dicho incumplimiento, incluida la capacidad para dictar normas legislativas. Estas medidas, adoptadas por la Federación en sustitución de un Land, tendrán una vigencia limitada ya que, al igual que sucede en el caso italiano, y sólo se mantendrán en tanto en cuanto el Land incumplidor adopta las medidas correspondientes para solucionar el incumplimiento ${ }^{53}$.

\section{V.4. BÉLGICA}

El Reino de Bélgica, que como se sabe es un Estado federal compuesto por tres comunidades, la francesa, la flamenca y la germanófona, y por tres regiones, la valona, la flamenca y la de Bruselas ${ }^{54}$; modificó en 1993 su Constitución para introducir en el art. 169 de Carta Magna belga la posibilidad de que las autoridades federales puedan sustituir a las entidades federadas cuando éstas no respeten los compromisos contraídos por el Estado $^{55}$. De tal modo que, si una Comunidad o Región incumpliera con el Derecho de la Unión Europea y por ello el Reino de Bélgica fuera condenado por el Tribunal de Justicia de la UE, el Estado podrá arrogarse las competencias de la entidad federada causante del incumplimiento para ejecutar el fallo de la sentencia condenatoria, en caso de que la entidad federada no cumpla con la sentencia. En su poder de sustitución las autoridades federales pueden llevar a cabo actividades tanto legislativas como ejecutivas pero éstas siempre deben estar circunscritas únicamente a la ejecución del fallo de la Sentencia; por otra parte, éstas medidas federales decaen cuando el ente federado «sustituido» lleva a cabo las actuaciones necesarias para poner fin al incumplimiento ${ }^{56}$.

52 Artículo 109.5 de la Ley Fundamental.

53 Apartado 5 del Artículo 23b de la Constitución Federal Austriaca.

54 Arts. 1,2, y 3 de la Constitución de Bélgica.

55 Artículo 169 de la Constitución de Bélgica: «A fin de garantizar el respeto de las obligaciones nacionales o supranacionales, los poderes citados en los artículos 36 y 37 (ejecutivo y legislativo federal) podrán, respetando las condiciones fijadas por la ley, sustituir temporalmente a los órganos aludidos en los artículos 115 y 121 (órganos de los entes federados). Tal ley deberá ser aprobada con la mayoría prevista en el artículo 4, último párrafo.» La Ley a la que hace mención este artículo es la Ley de 5 de mayo de 1993, especial sobre las relaciones internacionales de las Comunidades y Regiones, publicada en Moniteur Belge de 8 de mayo de 1993.

56 Informe, págs. 199-203. 


\section{PROPUESTAS DEL CONSEJO DE ESTADO}

El Consejo de Estado concluye su informe elevando una serie de propuestas relativas a cómo mejorar los mecanismos de prevención y reacción frente a incumplimientos del Derecho de la Unión Europea y, también, los procedimientos de repercusión de las sanciones impuestas.

\section{VI.1 CUESTIONES GENERALES RELATIVAS A LOS MECANISMOS DE PREVENCIÓN Y REACCIÓN}

Los mecanismos de prevención son aquellos que deben implementarse antes de que se constate la existencia de una violación del Derecho de la Unión Europea, mientras que los reactivos son aquellos que entran en funcionamiento cuando la violación del Derecho de la Unión ha sido constatada. Entiende el Consejo de Estado que existe violación constatada desde el momento en el que hay una sentencia del TJUE que haya declarado la existencia de incumplimiento ${ }^{57}$. No obstante, debe tenerse en cuenta que en las recomendaciones elaboradas por el Consejo de Estado se analizan las potencialidades tanto preventivas como reactivas de cada uno de los instrumentos propuestos.

\section{Cooperación y coordinación}

El mecanismo fundamental de prevención de incumplimientos que el Consejo de Estado propone es la coordinación y la cooperación entre el Estado central y las Comunidades Autónomas. Este mecanismo, sin embargo, no ofrece muchas potencialidades en el ámbito de la reacción ante un incumplimiento, pues quedaría únicamente circunscrito al procedimiento por incumplimiento ante el TJUE, agotándose, según indica el Consejo de Estado, con la eventual sentencia condenatoria que pudiera imponerse ${ }^{58}$.

El informe considera ${ }^{59}$ que los principales mecanismos de colaboración y cooperación se encuentran en el Acuerdo de 11 de diciembre de 1997 de la Conferencia para Asuntos Relacionados con las Comunidades Europeas, relativo a la participación de las Comunidades Autónomas en los procedimientos ante el Tribunal de Justicia de las Comunidades Europeas. Únicamente recomienda al respecto el Consejo de Estado que las reuniones preparatorias de la estrategia procesal ante el TJUE entre la Secretaría de Estado de la UE y la Comunidad sean preceptivas y no dependan de la voluntad de las partes para su realización ${ }^{60}$.

57 Informe, págs. 212-214. El Consejo de Estado se hace eco en su informe del debate doctrinal existente sobre el momento en el que debe instarse la reacción en tanto que existen aquellos que opinan que los mecanismos reactivos solo deben entrar en funcionamiento cuando medie Sentencia condenatoria.

58 Informe, pág. 224.

59 Vid. supra. II. Sobre el incumplimento del derecho de la Unión Europea y su procedimiento de sanción: implicación para las Comunidades Autónomas.

60 Informe, pág. 222. 


\section{Procedimientos ante el Tribunal Constitucional}

El Consejo de Estado sostiene que los procedimientos ante el Tribunal Constitucional no constituyen mecanismos idóneos para prevenir o reaccionar frente a incumplimientos de Derecho de la UE que tengan origen en una acción y omisión de una Comunidad Autónoma, debido por un lado, a lo breve de los plazos existentes para la interposición de recursos ante el Tribunal Constitucional y, por otro lado, a causa de la contradicción en que el Gobierno del Estado podría incurrir al instar un procedimiento contra una Comunidad Autónoma por considerar que ésta incumple con el Derecho Comunitario, mientras defiende al mismo tiempo ante las Instituciones Europeas la inexistencia de tal incumplimiento. Sin embargo, sí que considera el Consejo de Estado que, tanto el recurso de inconstitucionalidad como el conflicto negativo de competencias, ofrecen cierta operatividad para prevenir una declaración de incumplimiento por parte del TJUE. Así, el recurso de inconstitucionalidad podría conllevar la eliminación del ordenamiento jurídico nacional de una norma con rango de ley de una Comunidad Autónoma que fuera contraria al Derecho de la UE. Debe tenerse en cuenta que dicho incumplimiento debería ser invocado ante el Tribunal en relación con un incumplimiento constitucional susceptible de ser examinado por el Constitucional. Por su parte, el conflicto negativo podría servir para poner fin a un incumplimiento autonómico provocado por una inactividad ${ }^{61}$.

\section{VI.2. SOBRE LOS MECANISMOS DE PREVENCIÓN Y REACCIÓN FRENTE}

\section{A INCUMPLIMIENTOS NORMATIVOS}

Los incumplimientos normativos del Derecho de la UE son aquellos en los que se incurre bien cuando no se han adoptado las medidas legislativas o reglamentarias de desarrollo exigidas por una norma comunitaria, bien cuando se mantiene vigente en el ordenamiento nacional una norma contraria al Derecho de la Unión Europea. El Consejo de Estado recomienda tres mecanismos para prevenir y reaccionar frente a incumplimientos normativos del Derecho de la UE que pudieran tener su origen en una Comunidad Autónoma: la supletoriedad de las normas estatales, la utilización de leyes de armonización y la coerción estatal.

\section{La supletoriedad de las normas estatales}

A juicio del Consejo de Estado existe cierto margen para que se recurra a la supletoriedad del Derecho estatal a fin de asegurar el cumplimiento de las obligaciones del Derecho de la UE al amparo de lo establecido en el art. 149.1.3 de la Constitución. Además, en otros Estados, como por ejemplo en Italia, ha resultado ser un medio ciertamente eficaz para prevenir posibles incumplimientos por parte de las regiones, lo

61 Informe, págs. 225-228.

UNED. Teoría y Realidad Constitucional, núm. 28, 2011, pp. 609-632. 
que puede servir de referencia a España. La eficacia de este mecanismo depende, según el Consejo de Estado, del momento en que nos encontremos en referencia al posible incumplimiento; de modo que sería poco efectivo acudir a la supletoriedad una vez que ya exista Sentencia del TJUE en la que se constate el incumplimiento.

A la vista de lo anterior, el Consejo refiere en su informe dos recomendaciones al respecto del uso de la supletoriedad como mecanismo de prevención y reacción frente a incumplimientos del Derecho de la UE con origen en una Comunidad Autónoma. En primer lugar, es necesario que la norma estatal de naturaleza supletoria esté en vigor antes de que expiren los plazos establecidos en la norma comunitaria — normalmente el plazo de transposición de la directiva - para que exista una verdadera eficacia preventiva.Y, en segundo lugar, recomienda el Consejo que la norma estatal debería contener en todo caso indicaciones expresas de su naturaleza supletoria ${ }^{62}$.

\section{Las leyes de armonización}

Respecto al uso de las leyes de armonización previstas en el artículo 150.3 CE como instrumento de prevención y reacción frente a incumplimientos del Derecho de la UE, el Consejo de Estado sostiene que solo es idóneo en determinados casos y momentos. En primer lugar, señala el Informe que las leyes de armonización comparten cierta naturaleza con las directivas de la Unión, en tanto que estas últimas también tienen carácter armonizador; por eso solo se recomienda recurrir a ellas cuando sea necesario un plus de armonización a nivel estatal que no ofrezca la directiva. Este plus de armonización nacional que podría recomendar la utilización de una ley del artículo 150.3 puede venir demandado bien por la complejidad del desarrollo normativo que implica el desarrollo de una norma comunitaria, bien por la especial necesidad de una coherencia estatal de la legislación de desarrollo.

Por lo que se refiere al momento en que convendría recurrir a este mecanismo, el Consejo de Estado examina su eficacia como instrumento de prevención y reacción específicamente referido a la transposición de las directivas. Así, antes del plazo dado para la transposición, la ley de armonización podría evitar futuros incumplimientos, garantizando una adecuada trasposición de la normativa de la Unión por parte de las Comunidades Autónomas. Podría además servir para garantizar coherencia en la transposición de aquellas normativas comunitarias que afectaran a un significativo número de normas nacionales. Y por otra parte y directamente vinculado a lo anterior, también podría articularse, a través de dicha normativa de armonización, el sistema de notificación a la Comisión de las normas de transposición nacional operadas, en tanto que la no información la trasposición de directivas constituye ya de por sí un incumplimiento del Derecho de la Unión Europea fácilmente sancionable desde la entrada en vigor del Tratado de Lisboa.

Por lo que respecta al momento posterior al plazo de transposición, el Consejo de Estado considera dos escenarios distintos, con varias diversas posibilidades dentro de cada uno de ellos. El primero de los escenarios examinados se produce cuando la Comunidad

62 Informe, pág. 220. 
Autónoma no hubiera aún dictado normativa de desarrollo o trasposición; circunstancia esta en la que se examinan tres variantes.

En primer lugar, podría haber transcurrido el plazo de transposición sin que la Comunidad Autónoma hubiera transpuesto la normativa europea por considerar que la competencia para la transposición es del Estado, caso en el que, según el informe, debería llevar a cabo la transposición directamente el propio Estado. En segundo lugar, puede suceder que, una vez transcurrido el plazo de transposición, la Comunidad Autónoma no actúe en tanto que considere que la regulación ya existente — nacional y/o autonómicaes suficiente para dar por realizada la transposición que la norma europea demanda. Según el Consejo de Estado en este caso, y siempre que se advierta que es necesaria la transposición dada la insuficiencia de la normativa nacional, el Estado debería indicar a la Comunidad que su inactividad podría conllevar un incumplimiento del Derecho de la Unión. Y en tercer lugar, puede darse el caso de que la Comunidad Autónoma, aún considerándose competente para el desarrollo normativo de la legislación comunitaria en cuestión, no actúe; situación en la que el Informe desaconseja recurrir a la ley de armonización y acudir, en su caso, a una ley estatal de naturaleza supletoria.

El segundo de los escenarios tiene lugar cuando la Comunidad Autónoma ha procedido a dictar la normativa de desarrollo o transposición de la normativa comunitaria. En este caso el Consejo de Estado analiza dos situaciones. En primer lugar, que la normativa de la Comunidad Autónoma — que el Estado considera que podría conllevar un incumplimiento del Derecho de la Unión Europea - no haya sido aún declarada incompatible con la normativa comunitaria por la Unión. El Informe recomienda ante tal situación que solo se recurra a una ley estatal de armonización como mecanismo de prevención por razón de un significativo interés general, pues de otra forma podría interpretarse que la normativa estatal de armonización adolecería de una naturaleza de normativa impuesta a la Comunidad. Y en segundo lugar, si la normativa de la Comunidad Autónoma ya hubiera sido declara incompatible con el Derecho de la Unión por el Tribunal de Justicia de la Unión, entonces sí que, a juicio del Consejo de Estado, podría ser un recurso eficaz acudir a una ley estatal de armonización; en mayor medida si cabe, cuando dicha situación de incumplimiento autonómico se produjera o pudiera producirse en otras Comunidades Autónomas ${ }^{63}$.

\section{La coerción estatal}

El Informe examina también las potencialidades del artículo 155 CE como instrumento de prevención y reacción frente a incumplimientos de Derecho de la UE. El Consejo de Estado indica que el recurso a la coerción estatal previsto en el art. 155 CE debe únicamente reservarse para supuestos de naturaleza extraordinaria en los que una Comunidad Autónoma persista en un incumplimiento del Derecho del Unión declarado por el TJUE y se desprendan del mismo graves repercusiones para el conjunto del Estado. Recomienda el Consejo que los extremos relativos al procedimiento a seguir en

63 Informe, pág. 243-246. 
caso de utilización del artículo 155 sean regulados en una ley, en la que se contengan, además, los distintos mecanismos de garantía de cumplimiento del Derecho de la Unión Europea.

El Consejo de Estado también entiende que el artículo 155 podría ser utilizado para repercutir sobre la Comunidad Autónoma incumplidora la sanción que eventualmente recayera sobre España; recomendándose en su caso como un mecanismo subsidiario de repercusión de sanciones del mecanismo ordinario de repercusión.

\section{VI.3. SOBRE LOS MECANISMOS DE PREVENCIÓN Y REACCIÓN FRENTE A INCUMPLIMIENTOS EJECUTIVOS}

Por lo que los mecanismos de prevención y reacción se refiere en caso de incumplimientos ejecutivos, el Consejo de Estado sostiene que solamente debe considerarse que un incumplimiento de tal naturaleza se produce en tanto que una acción u omisión concreta de una Comunidad Autónoma ha sido efectivamente considerada como tal por el Tribunal de Justicia; pues de otra forma, si el Estado central actuara ex ante a la declaración del Tribunal de Justicia podría considerarse que éste trata de imponer una determinada actuación a la Comunidad Autónoma. Por este motivo, en el caso de incumplimientos ejecutivos, las posibles medidas de prevención, centradas en la coordinación y la colaboración, tendrían una eficacia limitada, según indica el informe.

Considera el Consejo de Estado que el mecanismo de reacción del Estado central ante los incumplimientos de naturaleza ejecutiva del Derecho de la Unión Europea por parte de una Comunidad Autónoma, debería ser el recurso contencioso administrativo. Sin embargo, al igual que ya se señalara en el caso de los recursos ante el Tribunal Constitucional, el Estado corre el peligro de caer en un efectiva contradicción si en el orden interno actúa judicialmente frente a la Comunidad Autónoma y el orden jurisdiccional europeo actúa en defensa de la actuación de la Comunidad para evitar una condena del Tribunal de Justicia.

Explora también el Consejo de Estado la posibilidad de que el Estado central, al amparo del art. $149.1 .3^{\circ} \mathrm{CE}$ en relación con el art. $93 \mathrm{CE}$, pudiera llevar a cabo una actuación destinada poner fin al incumplimiento ejecutivo de la Comunidad Autónoma. En este caso la actuación del Estado debería limitarse únicamente al ámbito de la Comunidad incumplidora. En cualquier caso dicha actuación estatal presentaría, además según indica el Informe - ciertos problemas de ejecución práctica como, por ejemplo, si el Estado central tiene o no habilitación para revisar de oficio la revisión de un acto ejecutivo de una Comunidad Autónoma. Finalmente, en aquellos casos en los que existiera una persistencia en un incumplimiento grave que conllevara significativas consecuencias para el interés general, el Consejo indica que sería recomendable recurrir a la coerción estatal, sobre la base del art. 155 de la Constitución, para poner fin a dicho incumplimiento ${ }^{64}$.

64 Informe, págs. 256-259. 


\section{VI.4. SOBRE LOS MECANISMOS DE REPERCUSIÓN DE SANCIONES}

El mecanismo de repercusión de sanaciones es el instrumento que permite al Estado central repetir, en todo o en parte, la sanción económica impuesta por el incumplimiento del ente de la Administración efectivamente responsable de dicho incumplimiento. Debe tenerse en cuenta que las previsiones contenidas en el informe al respecto de los mecanismos de repercusión han de examinarse a la luz del la Disposición Adicional Primera de la Ley de Economía Sostenible, que recientemente ha entrado en vigor — tal y como ya indicamos antes_ — ${ }^{65}$. En dicha disposición se han sentado las bases del procedimiento de repercusión general, que será desarrollado por el Gobierno mediante reglamento. Así dicha disposición ya contiene muchas de las previsiones y recomendaciones que el Consejo de Estado contiene en su informe. Se recomendaba, por ejemplo, la necesidad de establecer una definición amplia de las administraciones y entes sobre los cuales se podía repercutir la deuda, la necesidad de tipificar claramente que acciones u omisiones constituyen un incumplimiento del Derecho UE, regular los criterios de reparto de la sanción e introducir la posibilidad de un pago voluntario del ente incumplidor y, específicamente, de las Comunidades Autónomas. Todas estas recomendaciones serán, sin duda, tenidas en cuenta por el Gobierno a la hora de proceder a desarrollar reglamentariamente el mecanismo de repercusión ${ }^{66}$.

\section{VI.5. Sobre LA NECESIDAD DE UNA Ley para la GaRANTía DEL CUMPLIMIENTO Del Derecho de la Unión Europea}

La última de las propuestas contenidas en el Informe se refiere a la conveniencia de que se regulen conjuntamente en una Ley los mecanismos de prevención, reacción y en su caso repercusión relativos al incumplimiento del Derecho de la Unión Europea. Dicha

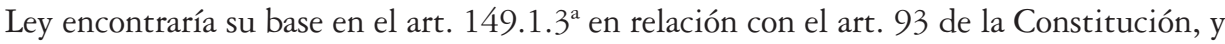
debería tener, según el Consejo de Estado, naturaleza de Ley ordinaria.

\section{CONCLUSIONES}

El Informe del Consejo de Estado constituye, por lo detallado y profuso de su análisis, una verdadera monografía de referencia respecto de la materia del incumplimiento del Derecho de la Unión Europea, en la que se analiza toda la problemática referida al misma y, muy específicamente, a los mecanismos de prevención, reacción y repercusión frente a incumplimientos del Derecho de la Unión con origen en las Comunidades Autónomas.

Por otra parte, y dado que este informe está llamado a suscitar no solo eventuales desarrollos normativos que lo tengan como referencia, sino también un debate en la doc-

65 Vid. supra apartado IV.1. Régimen General de repercusión de la responsabilidad: La Disposición Adicional Primera de la Ley de Economía Sostenible.

66 Informe, págs. 270-300. 
trina, no quisiera dejar de aportar aquí, al menos brevemente, dos reflexiones a propósito del mismo. La primera de ellas está dirigida a la conveniencia de que en una eventual futura reforma de la Constitución Española, y ante la ya más que urgente necesidad de su adaptación de la realidad europea, se procediera a introducir en la Carta Magna — en aras de una mayor seguridad jurídica tanto para el Estado como para las Comunidades Autónomas - algún tipo de previsión específicamente referida a los mecanismos de prevención, reacción y repercusión de los incumplimientos de Derecho de la Unión, tal y como ya ha sucedido en otros Estados miembros de naturaleza compuesta.

La segunda reflexión se orienta hacia la posibilidad de que el Derecho originario de la Unión Europea también fuera modificado en el sentido de establecer la posibilidad de que las Instituciones comunitarias puedan instar sanciones directamente contra los entes infraestatales de los Estados miembros efectivamente responsables de incumplimientos del Derecho de la UE, como ya la tiene, por ejemplo la Comisión para perseguir incumplimientos de ciertas normas de Derecho de la Unión Europea respecto de los sujetos particulares, o de los Estados. Cabe señalar que dicha reforma — en tanto que estos entes cuentan ya incluso con un órgano específico de representación de sus intereses en el marco institucional, el Comité de las Regiones; y se he previsto, además, que sus parlamentos puedan participar en el sistema de alerta temprana de control de la subsidiariedad-supondría, a mi juicio, un significativo y coherente paso en proceso de profundización de la integración europea. Dicho reconocimiento tendría una positiva repercusión en el avance de la integración europea federal multinivel, aunque no desconozco lo complejo de su posible articulación práctica.

$$
* * *
$$

TITLE: The Consultative Council Report of $15^{\text {th }}$ December 2010 abaut the non observance of EU Law enforcement.

ABSTRACT: This paper analyses the Consultative Council Inform of $15^{\text {th }}$ December 2010 about the inobservance of the EU Law caused by a Comunidad Autonoma and its effects. The Consultative Council examines all the possible mechanisms that could be used by the State to prevent and to act in case of a violation of EU Law.

RESUMEN: Este trabajo analiza el Informe del Consejo de Estado de 15 de diciembre de 2010 relativo a los mecanismos de prevención, reacción y repercusión ante el incumplimiento de Derecho de la Unión Europea provocado por una Comunidad Autónoma.

Palabras Clave: Incumplimiento de Derecho de la Unión Europed. España. Comunidades Autónomas. Prevención. Reacción. Repercusión de sanciones.

KeY WORDS: Violation of EU Law. Spain. Comunidad Autónoma. Prevention. Reaction. Penalty repercussion.

FeCHA DE RECEPCIÓN: 12.07.2011. FeCHA DE ACEPTACIÓN: 29.07.2011.

UNED. Teoría y Realidad Constitucional, núm. 28, 2011, pp. 609-632. 
